\title{
E-Learning, MALL and LMOOC or a New Didactic Tendency in Foreign Language Learning: A Meta-Analysis from 2014 to 2018
}

\author{
Toto Giusi Antonia, Limone Pierpaolo \\ Department of Humanities, Literature, Cultural Heritage, Education Sciences, Foggia, Italy \\ Email: giusy.toto@unifg.it
}

How to cite this paper: Antonia, T. G., \& Pierpaolo, L. (2020). E-Learning, MALL and LMOOC or a New Didactic Tendency in Foreign Language Learning: A Meta-Analysis from 2014 to 2018. Psychology, 11, 581-593. https://doi.org/10.4236/psych.2020.114039

Received: March 18, 2020

Accepted: April 17, 2020

Published: April 20, 2020

Copyright ( 2020 by author(s) and Scientific Research Publishing Inc. This work is licensed under the Creative Commons Attribution International License (CC BY 4.0).

http://creativecommons.org/licenses/by/4.0/

\section{(c) (i) Open Access}

\begin{abstract}
This meta-analysis explores the topic of formal and informal learning of foreign languages assisted by mobile devices, widely studied in international literature. The contemporary evolution of foreign language teaching shows itself in new forms such as m-learning, MALL and LMOOC. The increase of teaching/learning opportunities on e-learning platforms requires planning and didactical reflection to improve the chances of dialogue with the new generations of students. This paper analyzes the ongoing changes in terms o new pedagogy and instrumental models in the two five-year periods 2009-2013 and 2014-2018. This study adopts a meta-analysis approach to review the literature systematically, providing a complete analysis and synthesis of 156 studies, from 2014 to 2018. Previous studies focused on the massive introduction of mobile technology applied to the foreign language learning (FLL). The search in databases and citations was performed using keywords referring to FLL, MALL and LMOOC. Compared to existing reviews, the results confirm a skill-based approach to language learning with an emphasis on vocabulary, learning factors influencing students' perceptions and availability of new mobile technologies.
\end{abstract}

\section{Keywords}

MALL, M-Learning, LMOOC, Foreign Language Learning (FLL), Meta-Analysis

\section{Introduction}

Online learning can be more complex than traditional learning; in fact despite having common educational goals, they are experimenting with teaching, teaching and learning methodologies that are very different both in form and in con- 
tent. The first main revolution in online courses took place with the Massive Open Online Courses (MOOC), which provided online and open access courses on many disciplines. In 2012, most of the online courses concerned languages and information technology (Limone \& Pace, 2018). In the contemporary context, foreign language learning (FLL) has become more and more specialized to the point of offering many and various possibilities of study. In the last two years, the use of online courses has undergone further evolution so that some studies (Al-Emran et al., 2018) report students prefer to use mobile phones rather than other devices such as tablets or PCs when attending courses online. The last generation of courses for foreign languages can be divided into three types: 1) MOOC Language, specific online courses available through any digital medium, free but only at certain periods; 2) MALL, acronym that specifies that subset of mobile learning (m-learning) and finally; 3) CALL, that is computer-assisted language learning. Whereas MOOCs encourage interaction, collaboration and the creation of learning communities, MALLs simplify access to foreign language learning materials and allow students to communicate at any time with their teachers and colleagues. The analysis of the studies (Uzunboylu \& Genc, 2017) on foreign language learning courses on mobile devices highlights the tendency to focus on EFL, ESL, CLIL and EMI.

Literature review

Steps: A process of differentiation in parallel between m-learning and e-learning.

The plurality of forms assumed by online courses made the international scientific community ask itself about points of contact and differences among their existing types; today, according to Stockwell \& Hubbard (2013), e-learning does not evolve into m-learning, since the two models differ not only in the technological application support, but also in study tools and methodologies in the same way as MALL as an evolution on a simple technological basis of CALL. It seems better to differentiate among educational trends, user perspectives and learning outcomes in two models and systems. Over the last decade, a series of variations recorded in the scientific literature have proved how deep and epochal the changes have been in e-learning: until 2012 the discussions and studies focused almost exclusively on CALL and e-learning. Since 2013, the focus of research has shifted towards the study of MALL and e-learning. Starting from 2014, the definition of MOOC language learning (or foreign language learning) seems to characterize a specific type of quality course, more interested in user learning than in qualification certification (Bárcena \& Martín-Monje, 2014).

Meta-analysis: from e-learning to m-learning, from CALL to MALL and MOOC, different features?

Meta-analysis on the learning of foreign languages through e-learning, MALL and MOOC can be grouped into 3 directions (Table 1): m-learning applied to foreign languages (Elaish et al., 2017), trends in research design and application of mobile language learning (Hwang \& Fu, 2018) and results in terms of learning (Bravo et al., 2018). The meta-analysis on MALL and m-learning shows a similar 
Table 1. Meta analysis on MALL and m-learning applied to foreign language learning (2008-2017).

\begin{tabular}{ccc}
\hline Author & Dates covered & Number of papers \\
\hline Elaish et al. (2017) & $2010-2015$ & 88 \\
Bravo et al. (2018) & $2012-2017$ & 164 \\
Panagiotidis (2019) & $2013-2017$ & 38 \\
Hwang \& Fu (2018) & $2014-2017$ & 47 \\
\hline
\end{tabular}

pedagogical model to those separately conducted on MALL and m-learning that actually come to similar results in terms of learning. The Panagiotidis study (2019) reviews the MOOC studies on the foreign language learning. Elaish et al. (2017) identified the taxonomy and distribution of research, as well as the advantages and challenges providing recommendations to facilitate the learning and the effective use of the mobile English language. Educational data mining (EDM) combines data mining techniques with educational data for foreign languages to offer to students, instructors and researchers the knowledge needed to support academic processes (Corsi \& Rossi, 2018). Bravo et al. (2018) selected 208 documents, from which it emerged that the EDM is mainly used to predict student performance, to verify student motivation and to provide feedback to instructors. Although EDM has much to offer language students, it is not used to its full potential in education. Panagiotidis (2019) reported the results of $32 \mathrm{stu}-$ dies from the 2013-2017 five-year period and analyses the integration of the various media in MOOCs to learn foreign languages (defined either LMOOC or MOOLC) by providing references for assessment and certification of competences. Learning Analytics monitors learning styles and users results.

\section{Methodology}

\subsection{Research Goal}

This paper aims to outline current research trends in language learning assisted by mobile devices and mobile learning platforms and to highlight significant pedagogical implications by analyzing research from 2014 to 2018; the research approaches the qualitative meta-analysis using ground theory.

The following research questions were asked to see how the general framework of MALL research, m-learning and LMOOC applies to foreign language learning (2014-2018):

1) Which methodological tendencies, design and strategies are used to teach which language skills?

2) What are the learning and teaching contexts of the implementation of m-learning, MALL and MOOC?

3) What challenges and advantages do MALL, m-learning and MOOC produce in foreign language learning?

\subsection{Sample and Data Collection Procedure}

Qualitative coding and iterative categorizing traditionally used in the grounded 
theory (Strauss \& Corbin, 1997) tradition were used to synthesise and analyse data collected from selected papers through databases (e.g. Scopus, Psycinfo and Google Scholar), journals (e.g. Computer \& Education, Computer Assisted Language, Learning, System, Quarterly TESOL, Journal of Language Learning and Teaching, etc.) and searched for relevant and selected research citations. Due to the nature of the methodology, a (re) selection and continuous analysis were performed during which the coding took place. Conference proceedings were included, to avoid bias so as to be as unbiased as possible.

The sample included one hundred fifty-six papers met the criteria to form the data for the analysis. Keywords included the terms "foreign language mobile learning, MALL, language learning in m-learning, MOOC foreign language learning, LMOOC, mobile learning and language skills (speaking, reading, writing, listening, vocabulary and grammar)". Explicit coding, as stated by (Strauss \& Corbin, 1997), produced key codes such as LMOOC and MALL skills, technological tools, learning characteristics, research results, and limitations.

In the first stage, the present review included only papers with full-text availability. So conference abstracts, letters to the editor, opinion pieces and advertisements were excluded. In the second stage, we excluded papers whose titles or abstracts did not show any evidence of having investigated the use of digital media for foreign language learning. In the third stage, any paper not written in English was excluded. At the fourth stage, after a complete reading of the full texts of all included papers, we excluded those not reporting the use of m-learning, MALL or MOOC for foreign language learning. In the final stage, any duplicated paper was excluded. Finally, all the included papers were classified into two categories: applied and non-applied papers (to learning and to learner outcome). After the initial categorization, they were further classified according to their research objective. All the papers were summarized on the basis of key codes and content to be searched; We entered their data in tables, which include the source database, the author, the year of publication, the purpose, the problems in the research, the results, the target learners, the evaluation, the targeted linguistic competences and mobile language technologies used. The selection of these elements was in line with the objective and research questions of this review. Analysis (precoding, interim coding and final analysis) started at the end of this last stage.

\subsection{Sample Selection}

The search of all databases (2014-2018), showed 675 papers: 224 from Google Scholar, 101 from PsycInfo, 173 from Scopus, and 127 from WoS. Applying the inclusion criteria at the abstract level, 211 items were included. The full-text analysis involved 168 of those 211.12 papers turned out to be duplicated; therefore, the final number of the included papers was 156. As mentioned above, the included papers were categorized into two clusters: applied and non applied papers (Table 2 and Table 3). 67 papers concerned non-applied. Among these, 24 examined m-learning or MALL without any application, 4 papers covered the strategies and design used in both systems. 
Table 2. Results of meta-analysis.

\begin{tabular}{|c|c|c|}
\hline \multicolumn{3}{|c|}{$\begin{array}{l}\text { Total paper } \mathrm{n}=675 \\
\text { (224 from Google Scholar, } 101 \text { from PsycInfo, } 173 \text { from Scopus, and } 127 \text { from WoS) }\end{array}$} \\
\hline \multicolumn{2}{|c|}{ Inclusion criteria at the abstract level } & $\mathrm{N}=221$ items \\
\hline \multicolumn{2}{|r|}{ Full-text involved } & $\mathrm{N}=168$ \\
\hline \multicolumn{2}{|r|}{ Excluded duplicated } & $\mathrm{N}=156$ \\
\hline \multicolumn{3}{|c|}{ RESULTS $\mathrm{n}=156$} \\
\hline \multicolumn{2}{|r|}{$\begin{array}{l}\text { Applied papers } \\
\mathrm{n}=67\end{array}$} & $\begin{array}{c}\text { non applied papers } \\
\mathrm{n}=89\end{array}$ \\
\hline Type of papers & Topic & Number of papers $(n=67)$ \\
\hline \multicolumn{3}{|l|}{ Trends } \\
\hline Review & Application, system and projects & 11 \\
\hline Review & Methodology and theories. & 9 \\
\hline Survey papers & Previous usage of mobile applications and tools & 21 \\
\hline Paper & General pedagogy & 7 \\
\hline \multicolumn{3}{|c|}{ Design and strategy } \\
\hline Paper & instructional design & 6 \\
\hline Paper & App product design & 13 \\
\hline
\end{tabular}

The remaining 89 papers concerned the use of applications in the learning of the foreign language. Among these, the papers about pure mobile applications were 26 , whereas 37 papers concerned the mental abilities and the skills learned by the users and 26 papers studied assessment and certification.

\section{Results}

\subsection{The Non-Applied Papers}

The non-applied review was of two kinds, the first examined applications, systems or projects, the latter were general. The 21 survey papers (background) focused on the previous use of mobile applications and tools; 6 dealt with vocabulary acquisition and with writing and listening skills. A third classification consisted of 7 papers (theoretical studies) of general pedagogy including a model, a structure or a methodology for teaching of foreign languages in general. Two reviews of the aforementioned topics were included in this category. A final category of four papers concerned the design (2) and strategies (2) of applications that can improve students' learning outcomes (Table 3).

\subsection{The Applied Papers}

This section contains (Table 4), for the most part, linguistic research papers on learning a linguistic competence (vocabulary $n=7$, reading $n=3$, pronunciation $\mathrm{n}=4$, writing $\mathrm{n}=2$, on multiple skills $\mathrm{n}=3$ ). A subcategory (of learning games) concerns mobile gaming applications to improve linguistic performance, and 9 of the 12 selected papers specifically related to English learning. 
Table 4. The applied papers.

\begin{tabular}{ccc}
\hline Type of papers & Topic & Number of papers $(\mathrm{n}=89)$ \\
\hline Paper & Linguistic skills & 19 \\
Paper & Student performance & 18 \\
Paper & Learning games & $12(9)$ \\
Paper & Media & 14 \\
Paper & Assessment & 17 \\
Paper & Certification & 9 \\
\hline
\end{tabular}

Many papers ( $\mathrm{n}=18,11$ of which concerned how to solve problems of low motivation among students) throughout the period analyzed in this study examined the type of mobile devices to improve the performance of students into learning a foreign language (for content: student shows a complete and thorough knowledge of the subject. Explanations are well structured. Examples are provided, able to answer teacher's/examiner's questions. Student uses appropriate vocabulary. Grammar structures are correct or mostly correct (1 - 2 inaccuracies). Answers to questions relevant to the subject and are expanded (fluent speech). Motivation in language learning is a topic in itself, and it assumes a great variation between young, medium and older learners. E-learning will not solve motivation problems, but what has been found is a better connection to teens and young adults using these digital tools.

Finally, the media involved in the learning process concerned the use of Short Message Service and Multimedia Messaging Service to improve vocabulary ( $\mathrm{n}=$ 3 ), and the use of multimedia to improve language skills. An example is the use of music media $(n=7)$, for listening, images and voice (1) and for conversation (1). Two of the studies concerned the use of television in improving language skills. For the MOOC, specific themes concerned assessment $(n=17)$ and certification $(n=9)$.

\subsection{Distribution results}

In this section, we list our results by year of publication; Table 5 shows publication trends for each year in EU and ASIA (Table 4).

In general, the publication rate increased every year. Specifically, the application papers on the topic of m-learning become more frequent in the last two years.

In Table 6 specific abilities of foreign language learning have been categorized: vocabulary, reading, listening, speaking, writing or translating.

In 33 of 156 papers it was reported that their study covered all language skills, but some of them did not provide such information. Furthermore, 15 papers out of 89 focused on acquiring and improving vocabulary. A single study denounces the lack and need to study the effects of applying m-learning in the classroom. 
Table 5. Distribution of papers by year of publication.

\begin{tabular}{lc}
\hline Year & Number of publications \\
\hline 2018 & 40 \\
2017 & 35 \\
2016 & 34 \\
2015 & 29 \\
2014 & 18 \\
\hline
\end{tabular}

Table 6. Abilities of foreign language learning.

\begin{tabular}{cc}
\hline Linguistic abilities & n. paper \\
\hline Vocabulary & 15 \\
Reading & 3 \\
Listening & 7 \\
Speaking & 4 \\
Writing & 2 \\
Translating & 2 \\
\hline
\end{tabular}

\subsection{Distribution Results by Demographic Factors}

Most of the papers (134 of 156) studied the mobile learning of foreign languages among university students. In 106 papers the participants were of the same type, whereas about 28 have mixed participants, in most cases teachers and students together, although it should be noted that there are few studies just about teachers. The overwhelming majority of papers (99 out of 156) measure assessments in terms of learning of participants through tests, and according to the conclusions of the authors of the 149 papers reviewed, the use of m-learning, MOOC and MALL techniques improved the learning outcomes of users. The remaining papers showed no significant results.

\section{Discussion}

The present study reveals some interesting results, identifies some problems with existing research and classifies the existing literature on m-learning, LMOOC and MALL for the acquisition of foreign languages. The following sections report the results of a review based on the three research questions.

1) Which methodological tendencies, design and strategies are used to teach which language skills?

From the studied papers, it is clear that mobile applications are commonly used in informal learning and these applications had great development in formal education between 2014 and 2018 in MALLs and in the m-learning applied to foreign languages. The year 2014 marks the greatest diffusion of LMOOC, because there are 83 online courses in 16 e-learning platforms (Perifanou \& Economides, 2014). This study also represents the taxonomy of language skills 
aimed at learning foreign languages. Most of the papers investigate all language skills, but the greatest number concerned the acquisition and improvement of semantics, so that lexicon seems to be the most important skill to master (Hwang \& Fu, 2018). Vocabulary is not a skill, but rather an aspect of all language learning, as all e-learning is receptive skill based. Teachers use vocabulary almost exclusively at the lower levels. Vocabulary building is a classroom activity and influences the 4 skills (reading, listening, speaking and writing).

The design and strategies in these studies reflect the theories of social constructivism and social language. After all, second language learning is based on the acquisition of the first language. Design refers to educational design usually based on educational models of social and cognitive constructivism.

2) What are the learning and teaching contexts of the implementation of m-learning, MALL and MOOC?

The area of major study highlighted in this research is the success on the training plan in following online courses and the path of development and perseverance concluding an educational pathway started. The present study shows a common problem in e-learning studies, the general lack of needs diagnostic and the subsequent assessment of the effect of technologies on users (Ghanbarpour, 2017). Furthermore, the evaluation of students' performance takes place only through two real methods that field researchers can use: tests and surveys (interviews or observations could also be used).

Research, particularly action research has been taking a new look at evaluation. Many new techniques such as peer assessment or self assessment are now being explored. In language learning often the portfolio is being used in Europe. Participatory assessment (Sluijsmans et al., 2001) can stimulate the development of a competency-based curriculum, instead of generating what is otherwise known as inert learning, thus favouring the education of responsible and reflective students. Studies focus mainly on university students; there are no parallel studies on students and teachers. Moreover, with younger students, the use of $\mathrm{m}$-learning, LMOOC and MALL for the acquisition of foreign language has led to improvements. Therefore, research should focus on young students to have a clear perspective on m-learning in the classroom. Media and gamification are more likely to improve student outcomes and this may be due to immersive engagement during learning (Kukulska-Hulme \& Viberg, 2018). In the area of language learning, however, the use of e-learning technologies is very successful in communication but has little use in education. It seems clear that subsequent research will have to investigate the reasons that hinder this technology in the educational system.

3) What challenges and advantages do MALL, m-learning and MOOC produce in foreign language learning?

Among the advantages reported in the studies, there are improving encouragement of interaction and communication within an online learning community, training students to use an innovative didactic model and assessing whit al- 
ternative method that may be applied in their professional practices. This means that through innovative teaching strategies centred on the user and based on collaboration, such as peer learning and peer assessment, meaningful learning is promoted (Limone, 2012). M-learning is heavily used in informal learning; and through scientific experimentation, it tries to enter also into the formal area of education. The introduction of digital tools at school caused transformation and indicated new directions for didactic planning, (using bilingual dictionaries or language study software, playing, listening to the radio, sending text messages, etc.) because technologies created a connection with outside the school. In this context, the self-directed in learning and the collaborative learning are phenomena prompted by the use of technology, and they also produce autonomy in the learning of foreign languages. Students consider m-learning an intrusion into their lives outside of school (Limone \& Toto, 2018). E-learning has a positive effect on student involvement, which makes learning more enjoyable and motivates students to participate more proactively. Valuable tools for learning are the immediate feedback and availability of information on mobile devices, as they can develop the habit of self-learning (Monacis et al., 2016; Ponticorvo \& Miglino, 2018).

Among the disadvantages, research has narrowed the focus over the last 5 years (2014-2018), as the pervasiveness of mobile devices overcame the difficulties of use that were described in the studies between 2009-2013. Although attractive, m-learning is not yet a complete solution to the challenges of learning a foreign language. The opinions of students and teachers have negative perspectives on the future of e-learning due to concerns about quality and teaching related to the use of mobile devices in learning.

The sample refers that the main difficulties encountered concerning the disorientation for the first use of m-learning and the lack of training and the inability to concentrate before adapting to the new style of learning. A pre-set tool does not replace the feedback of teacher for some problems in learning (Toto et al., 2018), although peer learning in the last few years has made up for this difficulty.

The first studies on e-learning reported about difficulties in carrying out complex tests (Kukulska-Hulme \& Shield, 2008), today this can be overcome by a complex reflection on evaluation. For example, peer assessment testing gives important results in terms of learning. In general, although there are some papers, mobile language learning applications (MALL) lack the fundamentals of theory and methodology. M-learning applications are mainly designed by non-experts. Among the problems that may hamper learning, the small size of mobile devices, with poor presentations in a low audio-visual quality, emerges in the analysis of the first studies, an improved problem in the last phases of the e-learning experimentation (Kliuchko et al., 2015). Internet connection speed, reduced processing speed, limited space storage and mobile phone performance are complicated problems that can be solved only with the evolution of technology. Other concerns include socioeconomic access to technology for the under- 
privileged. Several experiments take place in the foreign language learning room, mobile applications cannot replace traditional classrooms yet. The meta-analyses also revealed many schools with significant geographical differences lack the tools and the innovative learning environments to learn foreign languages (hardware and software) and they also lack qualified professionals for L2 teaching.

\section{Limitations of the Study}

The first relevant limitation of this literature survey is the number and identity of source databases, although we believe that the chosen sample of sources could be a large and representative collection. Secondly, we confirm the fact that a comparison between the research activity on the theme of m-learning, LMOOC and MALL does not necessarily reflect the reality of its application, but simply the response of the research community to the trend. The study included also the publications of the first quarter of 2019.

\section{Conclusion}

The introduction of digital tools in formal education has produced a revolution in traditional teaching and learning models. The experimentation associated with learning foreign languages represents a major challenge for students and teachers. The purpose of this study is to provide a complete analysis of the existing literature to understand the evolution of theoretical trends, the design and the strategies used to teach, which language skills and what are the learning and teaching contexts of the implementation of m-learning, LMOOC and MALL. Finally, it presents the advantages and challenges of foreign language learning and therefore researches the best strategies to achieve valid conclusions (Perifanou, 2016; Mazza et al., 2018).

Keywords used in the research may not cover all relevant areas, but the research represents the use of previous studies on mobile learning foreign languages. The expansion of the keywords with the LMOOC research area has allowed for a retrieval of more studies. The results of the systematic review could provide educators and researchers with a comprehensive view of research trends in the use of m-learning, MOOC and MALL in foreign language learning.

For delineating the research trends on FLL, this research has shown an orientation based on the skills towards language education mediated by mobile devices. As far as LMOOCs are concerned, research is oriented towards the themes of assessment and certification of qualifications. This problem is also common to the other disciplines taught in MOOCs. Liu \& Brantmeier (2019), who study young EFL learners, point out that if the tasks of mobile learning are not compulsory, students are reluctant to be involved in these tasks. Therefore, e-learning activities must be integrated into traditional learning; otherwise they will not be successful, if based on the spontaneity of the study. Studies of FLL are more focused on the research of European foreign languages and the growing interest 
and enthusiasm of teachers and students is considered a rich sector to be explored in future research also for its intercultural nature (Nardi \& Ranieri, 2019).

The use of technologies within traditional teaching has oriented the learning process towards the collaborative construction of knowledge, from exploratory learning and problem-based training. The active part required of the students allows him to overcome the hegemony of digital tools, which become mediators to carry out activities, manipulate objects and construct meanings. The Eurobarometer "the Europeans and their languages" (2012) describe as fundamental the motivation in the language learning process, which is in turn influenced by the family situation, the level of education and the social condition. Literacy becomes a process that encompasses (multiple) languages and multiple (even digital) skills. Specifically for foreign language learning, some limitations to these matters concern vocabulary difficulties and the lack of reading. Also the area of disability related to this specific learning is completely ignored in the studies. Future research should focus on this last topic and examine whether variables such as gender that may influence the quality of student participation in m-learning (Rivoltella, 2008).

In conclusion, this study reviews the research published in recent years regarding e-learning, foreign language learning courses on mobile devices highlights the tendency to focus on EFL, ESL, CLIL and EMI, and reach students in economic difficulty that could be excluded from the educational process (Toto, 2018), the use of such technologies can support the traditional lesson and its learning objectives also in language learning.

The evolution of e-learning for FLL present today in the forms of m-learning, MALL and LMOOC is developing in a multiplicity of training offer and of new forms of assessment and certification that need further study and in-depth analysis.

\section{Conflicts of Interest}

The authors declare no conflicts of interest regarding the publication of this paper.

\section{References}

Al-Emran, M., Mezhuyev, V., \& Kamaludin, A. (2018). Technology Acceptance Model in M-Learning Context: A Systematic Review. Computers \& Education, 125, 389-412. https://doi.org/10.1016/j.compedu.2018.06.008

Bárcena, E., \& Martín-Monje, E. (2014). 1 Introduction. Language MOOCs: An Emerging Field. In Language MOOCs (pp. 1-15). Sciendo Migration. https://doi.org/10.2478/9783110420067.1

Bravo, J., Frances, B., \& Isaac, C. S. (2018). Data Mining in Foreign Language Learning. Wiley Interdisciplinary Reviews: Data Mining and Knowledge Discovery.

Corsi, M., \& Rossi, P. G. (2018). Rethinking the Design Process, Rethinking the Curriculum. Education Sciences \& Society, 8, No. 2. https://doi.org/10.3280/ess2-2017oa5733

Elaish, M. M., Shuib, L., Ghani, N. A., Yadegaridehkordi, E., \& Alaa, M. (2017). Mobile 
Learning for English Language Acquisition: Taxonomy, Challenges, and Recommendations. IEEE Access, 5, 19033-19047. https://doi.org/10.1109/ACCESS.2017.2749541

Ghanbarpour, M. (2017). A Qualitative Meta-Synthesis of Research on Dynamic Assessment of Second/Foreign Language Learning: Implications for Language Teachers. Journal of Language Teaching and Research, 8, 731. https://doi.org/10.17507/jltr.0804.12

Hwang, G. J., \& Fu, Q. K. (2018). Trends in the Research Design and Application of Mobile Language Learning: A Review of 2007-2016 Publications in Selected SSCI Journals. Interactive Learning Environments, 27, 567-581. https://doi.org/10.1080/10494820.2018.1486861

Kliuchko, M., Heinonen-Guzejev, M., Monacis, L., Gold, B. P., Heikkilä, K. V., Spinosa, V., Tervaniemi, M., \& Brattico, E. (2015). The Association of Noise Sensitivity with Music Listening, Training, and Aptitude. Noise and Health, 17, 350-357. https://doi.org/10.4103/1463-1741.165065

Kukulska-Hulme, A., \& Shield, L. (2008). An Overview of Mobile Assisted Language Learning: From Content Delivery to Supported Collaboration and Interaction. Recall, 20, 271-289. https://doi.org/10.1017/S0958344008000335

Kukulska-Hulme, A., \& Viberg, O. (2018). Mobile Collaborative Language Learning: State of the Art. British Journal of Educational Technology, 49, 207-218. https://doi.org/10.1111/bjet.12580

Limone, P. (2012). Ambienti di apprendimento e progettazione didattica. Proposte per un sistema educativo transmediale (pp. 1-176). Carocci.

Limone, P., \& Pace, R. (2018). Teacher Training and Digital Paths. Revolution in the School: A Project for Lifelong Learning. In Teacher Training and Professional Development: Concepts, Methodologies, Tools, and Application (pp. 657-675). New York: IGI Global. https://doi.org/10.4018/978-1-5225-5631-2.ch029

Limone, P., \& Toto, G. A. (2018). The Psychological Constructs and Dimensions Applied to Sports Performance: A Change of Theoretical Paradigms. Journal of Physical Education and Sport, 18, 2034-2038.

Liu, H., \& Brantmeier, C. (2019). "I know English”: Self-Assessment of Foreign Language Reading and Writing Abilities among Young Chinese Learners of English. System, 80, 60-72. https://doi.org/10.1016/j.system.2018.10.013

Mazza, S., Ligorio, M. B., \& Cacciamani, S. (2018). Instrumental Orchestration for the Insertion of "Virtual Classrooms" at School. Qwerty-Open and Interdisciplinary Journal of Technology, Culture and Education, 13, 49-65.

Monacis, L., de Palo, V., Sinatra, M., \& Berzonsky, M. D. (2016). The Revised Identity Style Inventory: Factor Structure and Validity in Italian Speaking Students. Frontiers in Psychology, 7, Article No. 883. https://doi.org/10.3389/fpsyg.2016.00883

Nardi, A., \& Ranieri, M. (2019). Comparing Paper-Based and Electronic Multiple-Choice Examinations with Personal Devices: Impact on Students' Performance, Self-Efficacy and Satisfaction. British Journal of Educational Technology, 50, 1495-1506. https://doi.org/10.1111/bjet.12644

Panagiotidis, P. (2019). MOOCs for Language Learning. Reality and Prospects. In Society for Information Technology \& Teacher Education International Conference (pp. 286-292). Association for the Advancement of Computing in Education (AACE).

Perifanou, M. (2016). Worldwide State of Language MOOCs (pp. 386-390). CALL Communities and Culture-EUROCALL.

https://doi.org/10.14705/rpnet.2016.eurocall2016.593 
Perifanou, M., \& Economides, A. (2014). MOOCs for Foreign Language Learning: An Effort to Explore and Evaluate the First Practices. In Proceedings of INTED 2014 Conference (pp. 3561-3570). Valencia, Spain.

Ponticorvo, M., \& Miglino, O. (2018). Hyper Activity Books for Children: How Technology Can Open Books to Multisensory Learning, Narration and Assessment. Qwerty-Open and Interdisciplinary Journal of Technology, Culture and Education, 13, 46-61.

Rivoltella, P. C. (2008). Digital Literacy: Tools and Methodologies for Information Society: Tools and Methodologies for Information Society. New York: IGI Global. https://doi.org/10.4018/978-1-59904-798-0

Sluijsmans, D. M., Moerkerke, G., Van Merrienboer, J. J., \& Dochy, F. J. (2001). Peer Assessment in Problem Based Learning. Studies in Educational Evaluation, 27, 153-173. https://doi.org/10.1016/S0191-491X(01)00019-0

Stockwell, G., \& Hubbard, P. (2013). Some Emerging Principles for Mobile-Assisted Language Learning. Monterey, CA: The International Research Foundation for English Language Education.

http://www.tirfonline.org/wp-content/uploads/2013/11/TIRF_MALL_Papers_Stockwel lHubbard.pdf

Strauss, A., \& Corbin, J. M. (1997). Grounded Theory in Practice. London: Sage.

Toto, G. A. (2018). From Educational Contexts to Addictions: The Role of Technology in Teaching Methodologies and in Prevention as an Educational Function. Journal of e-Learing and Knowledge Society, 14, 203-212.

Toto, G. A., Ruberto V., \& Toto, M. (2018). Technologies: A Comparison between Negative Aspects of Addictions and Effects on Text Comprehension. In Proceedings of the 31th International Business Information Management Association Conference-IBIMA (pp. 6380-6390). Milan, Italy.

Uzunboylu, H., \& Genc, Z. (2017). Analysis of Documents Published in Scopus Database on Foreign Language Learning Through Mobile Learning: A Content Analysis. Profile Issues in Teachers' Professional Development, 19, 99-107.

https://doi.org/10.15446/profile.v19n_sup1.68624 